\title{
Influence of voltine ecotype divergence on genetic and haplotype variation in the Asian corn borer
}

\author{
Yangzhou Wang ${ }^{1}$, Kyung Seok Kim² ${ }^{2}$, Qiyun Li $^{1}$, Yunyue Zhang ${ }^{1}$, Zhen-Ying Wang ${ }^{3}$, and \\ BRAD COATES ${ }^{4}$
}

\author{
${ }^{1}$ Jilin Academy of Agricultural Sciences \\ ${ }^{2}$ Iowa State University \\ ${ }^{3}$ Chinese Academy of Sciences \\ ${ }^{4} \mathrm{USDA}$ ARS
}

October 1, 2020

\begin{abstract}
Diapause is an adaptive dormancy strategy by which arthropods endure extended periods of adverse climatic conditions. Seasonal variation in larval diapause initiation and duration in Ostrinia furnacalis influences adult mating generation number (voltinism) across different local environments. The degree of mating period overlap between sympatric voltine ecotypes influence hybridization level, but impacts on $O$. furnacalis population genetic structure and evolution of divergent adaptive phenotypes remains uncertain. Genetic differentiation was estimated between voltine ecotypes collected from 8 locations. Mitochondrial haplotypes were significantly different between historically allopatric univoltine and bivoltine locations. Haplotypes from sympatric locations were clustered more-closely to bivoltine locations, but influenced by local demographics. Additionally, analyses of single nucleotide polymorphism (SNP) genotypes implicate voltinism, as opposed to geographic distance, as contributing to low, but significant levels of variation among voltine ecotypes. Regardless, only 11 of 257 SNP loci were predicted to be under selection, suggesting population genetic homogenization except at loci proximal to factors putatively responsible for locally adaptive or voltinism-specific traits. These findings provide evidence that divergent voltine ecotypes may be maintained in allopatric and sympatric areas despite relatively high rates of nuclear gene flow, yet influence of voltinism on maintenance of observed haplotype divergence remains unresolved.
\end{abstract}

\section{Hosted file}

Manuscript_EvolutionSubmission.DOCX available at https://authorea.com/users/295943/articles/ 484301-influence-of-voltine-ecotype-divergence-on-genetic-and-haplotype-variation-inthe-asian-corn-borer 